\title{
On the Solutions of the Lorentz-Dirac Equation
}

\author{
D. Vogt* \\ Instituto de Física Gleb Wataghin, Universidade Estadual de Campinas \\ 13083-970 Campinas, S.P., Brazil \\ P. S. Letelier ${ }^{\dagger}$ \\ Departamento de Matemática Aplicada-IMECC, Universidade Estadual \\ de Campinas 13083-970 Campinas, S.P., Brazil
}

\begin{abstract}
We discuss the unstable character of the solutions of the Lorentz-Dirac equation and stress the need of methods like order reduction to derive a physically acceptable equation of motion. The discussion is illustrated with the paradigmatic example of the non-relativistic harmonic oscillator with radiation reaction. We also illustrate removal of the noncasual pre-acceleration with the introduction of a small correction in the Lorentz-Dirac equation.

PACS: 41.60.-m, 02.60.Cb

Keywords: Lorentz-Dirac equation, radiation reaction, stability, harmonic oscillator.
\end{abstract}

The classical equation of motion of a point charge including radiation reaction is the

*e-mail: danielvt@ifi.unicamp.br

†e-mail: letelier@ime.unicamp.br
Lorentz-Dirac (LD) equation [1],

$$
\ddot{x}^{\mu}=f^{\mu}+b\left(\dddot{x}^{\mu}+\frac{\dot{x}^{\mu}}{c^{2}} \ddot{x}^{\nu} \ddot{x}_{\nu}\right)
$$

where a dot indicates derivative with respect to the proper time $\tau ; f^{\mu}$ is the external 4 -force per unit mass and $b=\frac{2 e^{2}}{3 m c^{3}}$. Although the LD equation is based in solid physical ground (Special Relativity and Electrodynamics) it has some unusual mathematical properties that put some doubts about the suitability of this equation to represent the motion equation of a charged particle with radiation reaction. Since the LD equation is a third order ordinary differential equation, not only the initial position and velocity, but also the initial acceleration must be given to specify a unique solution. Moreover, one has to determine with an infinity accuracy the initial value of the acceleration that eliminates the part of the solutions that grow exponentially in time ("runaway" solution). 
Recently, Spohn [2] found that the physical solutions of the LD equation lie on a critical manifold in phase space that consists exclusively of repelling fixed points. On this critical surface the motion is free of runaway solutions and is governed by an effective second order equation. If an initial condition slightly off the critical surface is given, the solution grows exponentially fast. This lack of stability of solutions with respect to small variations of initial conditions means that the LD equation does not represent a mathematically well posed problem [3] and in consequence its applicability is limited.

Difficulties also arise in the numerical solution of the LD equation 44. Even if one knows the correct initial value of the acceleration, in the usual forward numerical integration the runaway contributions to the solution grow extremely fast due to numerical errors. As an alternative, Aguirregabiria [5] proposes a method of order reduction in which Eq.(11) is substituted by a second order equation of motion with no runaway solutions. The explicit form of this second order equation cannot be obtained in general, so a series of successive approximations $\ddot{x}^{\mu}=\xi_{n}^{\mu}$, $(n=0,1, \ldots)$ is constructed:

$$
\begin{aligned}
\ddot{x}^{\mu} & =\xi_{0}^{\mu}=f^{\mu} \\
\ddot{x}^{\mu} & =\xi_{1}^{\mu}=f^{\mu}+b\left(\frac{\partial f^{\mu}}{\partial \tau}+\frac{\partial f^{\mu}}{\partial x^{\nu}} \dot{x}^{\nu}+\frac{\partial f^{\mu}}{\partial \dot{x}^{\nu}} f^{\nu}\right. \\
& \left.+\dot{x}^{\mu} \frac{f^{\nu} f_{\nu}}{c^{2}}\right), \\
\ddot{x}^{\mu} & =\xi_{n+1}^{\mu}=f^{\mu}+b\left(\frac{\partial \xi_{n}^{\mu}}{\partial \tau}+\frac{\partial \xi_{n}^{\mu}}{\partial x^{\nu}} \dot{x}^{\nu}+\frac{\partial \xi_{n}^{\mu}}{\partial \dot{x}^{\nu}} \xi_{n}^{\nu}\right. \\
& \left.+\dot{x}^{\mu} \frac{\xi_{n}^{\nu} \xi_{n \nu}}{c^{2}}\right) .
\end{aligned}
$$

We note that in this approach only the usual initial conditions (position and velocity) are required and the equations for a finite $n$ are stable with respect to variations of initial conditions.

Rohrlich [6], inspired on Landau [7], argues that the original LD equation should be replaced by Eq.(3) as the exact equation of motion of a radiating point particle, since it is of second order, satisfies the principle of inertia, and the radiation reaction term vanishes in the absence of an external force. This prescription is based on physical arguments, but only the confrontation with experimental results will establish its validity. For proposals of experimental tests, see [2].

The purpose of this letter is to illustrate the above discussion with the significant example of the non-relativistic one-dimensional harmonic oscillator with radiation reaction. This problem has an analytical solution that can be used to construct the effective second order equation of motion on the above mentioned critical surface. The successive approximations scheme (2)-(44) and solutions 
from direct numerical integration of the nonrelativistic LD equation can be confronted with the exact solution. The study of this example will show us the limitations of the LD equation

to describe the motion of a charge particle interacting with its own radiation (back reaction). Also we shall discuss the addition of a small correction term that eliminates the preacceleration in the solutions to the LD equation [8]. In particular we discuss, in some detail, the case of a constant force that acts on the charge particle during a finite time.

The one-dimensional non-relativistic motion of an electron subjected to a harmonic force is governed by the Abraham-Lorentz limit of the LD equation,

$$
\frac{d^{2} x}{d t^{2}}=-\omega^{2} x+b \frac{d^{3} x}{d t^{3}},
$$

whose exact solution is given by $x(t)=c_{1} e^{\alpha_{1} t}+e^{\alpha_{2 r} t}\left(c_{2} \cos \left(\alpha_{2 i} t\right)+c_{3} \sin \left(\alpha_{2 i} t\right)\right)$.

The constants $c_{1}, c_{2}$ and $c_{3}$ depend on the initial conditions and

$$
\begin{gathered}
\alpha_{1}=\frac{1}{3 b}\left(1+\beta^{1 / 3}+\beta^{-1 / 3}\right), \\
\alpha_{2 r}=\frac{1}{3 b}\left(1-\frac{\gamma^{1 / 3}}{4}-\gamma^{-1 / 3}\right), \\
\alpha_{2 i}=\frac{\sqrt{3}}{6 b}\left(\beta^{1 / 3}-\beta^{-1 / 3}\right), \\
\beta=1+\frac{27}{2} \omega^{2} b^{2}+3 \sqrt{3} \omega b \sqrt{1+\frac{27}{4} \omega^{2} b^{2}}, \\
\gamma=8+108 \omega^{2} b^{2}+12 \sqrt{3} \omega b \sqrt{4+27 \omega^{2} b^{2}} .
\end{gathered}
$$

¿From Eq. (6) we find the expressions for the velocity and acceleration:

$$
\begin{gathered}
\dot{x}(t)=\alpha_{1} c_{1} e^{\alpha_{1} t}+\left(c_{2} \alpha_{2 r}+c_{3} \alpha_{2 i}\right) e^{\alpha_{2 r} t} \cos \left(\alpha_{2 i} t\right) \\
+\left(c_{3} \alpha_{2 r}-c_{2} \alpha_{2 i}\right) e^{\alpha_{2 r} t} \sin \left(\alpha_{2 i} t\right) \\
\ddot{x}(t)=\alpha_{1}^{2} c_{1} e^{\alpha_{1} t}+\left[c_{2}\left(\alpha_{2 r}^{2}-\alpha_{2 i}^{2}\right)+2 c_{3} \alpha_{2 r} \alpha_{2 i}\right] \times \\
e^{\alpha_{2 r} t} \cos \left(\alpha_{2 i} t\right)+\left[c_{3}\left(\alpha_{2 r}^{2}-\alpha_{2 i}^{2}\right)\right. \\
\left.\quad-2 c_{2} \alpha_{2 r} \alpha_{2 i}\right] e^{\alpha_{2 r} t} \sin \left(\alpha_{2 i} t\right)
\end{gathered}
$$

In terms of the initial conditions $x(0)=x_{0}$, $\dot{x}(0)=\dot{x}_{0}$ and $\ddot{x}(0)=\ddot{x}_{0}$, the constants $c_{1}, c_{2}$ and $c_{3}$ are given by

$$
\begin{gathered}
c_{1}=\frac{x_{0}\left(\alpha_{2 r}^{2}+\alpha_{2 i}^{2}\right)+\ddot{x}_{0}-2 \alpha_{2 r} \dot{x}_{0}}{\left(\alpha_{1}-\alpha_{2 r}\right)^{2}+\alpha_{2 i}^{2}} \\
c_{2}=\frac{x_{0} \alpha_{1}\left(\alpha_{1}-2 \alpha_{2 r}\right)-\ddot{x}_{0}+2 \alpha_{2 r} \dot{x}_{0}}{\left(\alpha_{1}-\alpha_{2 r}\right)^{2}+\alpha_{2 i}^{2}}, \\
c_{3}=-\left[x_{0} \alpha_{1}\left(\alpha_{1} \alpha_{2 r}-\alpha_{2 r}^{2}+\alpha_{2 i}^{2}\right)+\right. \\
\left.+\ddot{x}_{0}\left(\alpha_{1}-\alpha_{2 r}\right)+\dot{x}_{0}\left(\alpha_{2 r}^{2}-\alpha_{1}^{2}-\alpha_{2 i}^{2}\right)\right] \\
\alpha_{2 i}\left[\left(\alpha_{1}-\alpha_{2 r}\right)^{2}+\alpha_{2 i}^{2}\right] .
\end{gathered}
$$

Using Eq.(6) and Eq.(12), Eq.(13) can be cast as

$$
\begin{aligned}
\ddot{x}(t)=c_{1}\left[\left(\alpha_{1}-\alpha_{2 r}\right)^{2}\right. & \left.+\alpha_{2 i}^{2}\right] e^{\alpha_{1} t}+2 \alpha_{2 r} \dot{x} \\
& -\left(\alpha_{2 r}^{2}+\alpha_{2 i}^{2}\right) x .
\end{aligned}
$$

Since $\alpha_{1}>0$, the term $e^{\alpha_{1} t}$ has a runaway character, so we must set $c_{1}=0$ to eliminate the unphysical solutions. Then Eq.(17) takes the form

$$
\ddot{x}(t)=2 \alpha_{2 r} \dot{x}-\left(\alpha_{2 r}^{2}+\alpha_{2 i}^{2}\right) x .
$$

This is the equation of motion of a damped harmonic oscillator and represents the effective second order equation on the critical surface. In this particular problem the effective equation can be obtained exactly. 


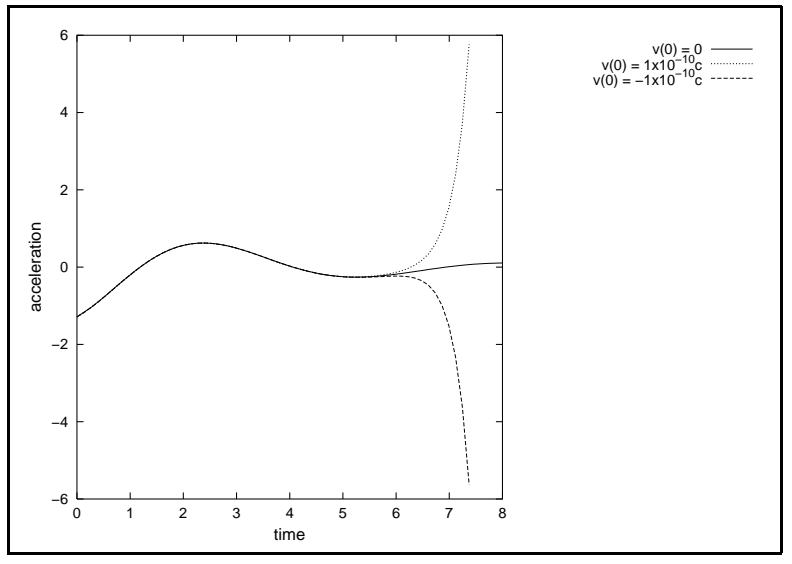

Figure 1: Acceleration as function of time with $b \omega=2 \pi / 7$ and initial conditions $x(0)=$ $1, \dot{x}(0)=0$ (solid curve), $\dot{x}(0)= \pm 1 \times 10^{-10} \mathrm{c}$ (dashed curves) and $\ddot{x}(0)$ given by Eq. (18). The curves were ploted using the analytical solutions of Eq.(15) (1 unit of length $=$ $2.818 \times 10^{-15} \mathrm{~m}, 1$ unit of time $=9.399 \times 10^{-24}$ s).

The scheme of successive approximations applied to Eq.(15) results in equations similar to Eq.(18) [5]

$$
\ddot{x}=-\gamma_{n} \dot{x}-\omega_{n}^{2} x, n=0,1, \ldots
$$

where the coefficients satisfy the recurrence relations

$$
\begin{gathered}
\gamma_{0}=0, \\
\omega_{n+1}^{2}=\omega_{0}^{2}-b \omega_{n}^{2} \gamma_{n}, \\
\gamma_{n+1}=b\left(\omega_{n}^{2}-\gamma_{n}^{2}\right) .
\end{gathered}
$$

When $b \omega \lesssim 0.95$ these successive approximations will always converge.

Figure 1 shows the curves of acceleration as function of time with $x(0)=1, b \omega=2 \pi / 7$

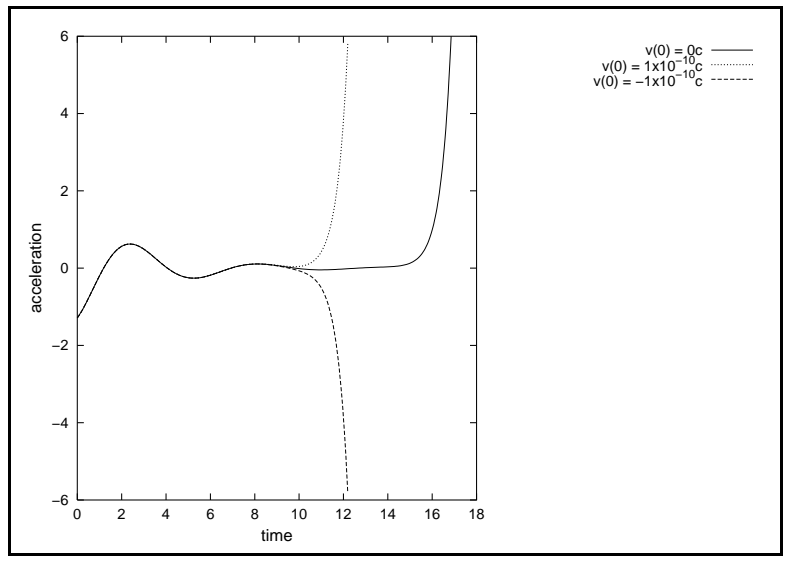

Figure 2: Acceleration as function of time with $b \omega=2 \pi / 7$ and initial conditions $x(0)=$ 1 unit of length, $\dot{x}(0)=0$ (solid curve), $\dot{x}(0)= \pm 1 \times 10^{-10} \mathrm{c}$ (dashed curves) and $\ddot{x}(0)$ given by Eq.(18). The curves were drawn numerically integrating Eq. (15) (1 unit of length $=2.818 \times 10^{-15} \mathrm{~m}, 1$ unit of time $\left.=9.399 \times 10^{-24} \mathrm{~s}\right)$.

(our units are: 1 unit of length $=2.818 \times$ $10^{-15} \mathrm{~m}, 1$ unit of time $=9.399 \times 10^{-24}$ s). The continuous curve was obtained from Eq.(18) with initial velocity $\dot{x}(0)=0$, the dashed curves with the same initial values for position and acceleration, but with $\dot{x}(0)=$ $\pm 1 \times 10^{-10} \mathrm{c}$ and Eq. (13) - (16). This is equivalent to take initial conditions slightly off the critical surface. The acceleration diverges after a few units of time, indicating the instability of the critical surface.

Figure 2 shows the curves of acceleration as function of time with $b \omega=2 \pi / 7$ and the same initial conditions as in figure 1, but now Eq.(5) was solved numerically (fourthfifth order Runge-Kutta with adaptive step algorithm). Even though the value of the ini- 


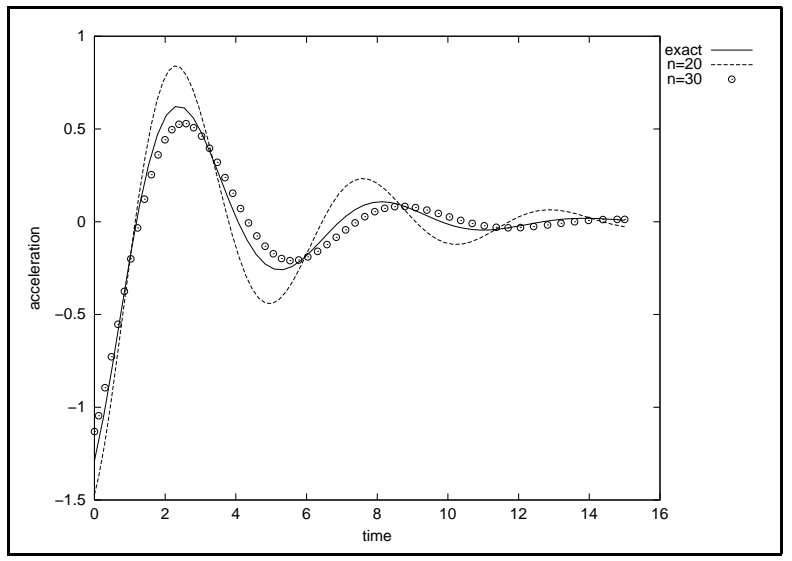

Figure 3: Acceleration as function of time with $b \omega=2 \pi / 7$ and initial condition $x(0)=1$ unit of length and $\dot{x}(0)=0$. The curves were plot using successive approximations Eq.(19) for $n=20$ and $n=30$ ( 1 unit of length = $2.818 \times 10^{-15} \mathrm{~m}, 1$ unit of time $=9.399 \times 10^{-24}$ s).

tial acceleration given by Eq.(18) is used with 16 significant figures, the runaway contributions make also the numerical solution useless after a few time units.

Finally, in Figure 3 the solutions calculated with the successive approximations (19) are compared with the exact solution using initial conditions $x(0)=1$ and $\dot{x}(0)=0$. Although the convergence is slow, no instability is seen.

This example clarifies the uselessness of Eq.(11) in practice. Even though, for very special cases, some exact solutions of the LD equation are known 9] for actual situations one must rely on numerical solutions. But, to eliminate the runaway components one would have to know the exact initial conditions and to perform the computations with infinite precision. The knowledge of the ex- act initial conditions imposes a physically impossible condition to be achieved. Also, do numerics with infinite precision is not possible. Recently, Chicone et al [10] pointed out that high order equations like Eq.(1) must be viewed as an intermediate step in the derivation of the physically correct, second order equation. A possible method to find this equations is the use of successive approximations. However, there is no guarantee of convergence.

We would like to comment another unusual feature of the LD equation, the phenomenon of pre-acceleration. When the external force is an explicit function of time, the solution of the one-dimensional non-relativistic version of Eq.(11) can be written as 9

$$
\ddot{x}(t)=\frac{1}{b} \int_{t}^{\infty} e^{-\left(t^{\prime}-t\right) / b} f\left(t^{\prime}\right) \mathrm{d} t^{\prime} .
$$

It follows from Eq.(21) that a nonzero acceleration exists before the external force is applied at $t=0$, thus violating cusality. In a nice monograph on the LD equation, Yaghjian 8 carefully rederives the equation of motion for the extended model of the electron as a charged insulating sphere of radius $a$. He shows that the multiplication of the electromagnetic self force by a correction function $\eta(\tau)$ eliminates the pre-acceleration from the solution to the original LD equation without introducing false discontinuities in velocity across $\tau=0$ or spurious delta functions and their derivatives at $\tau=0$. The correction function increases monotonically from zero to one in the time it takes light to travel across the electron, and it approaches zero like $\tau^{2}$ or faster as $\tau$ approaches zero from 
the positive (right) side. This modification is needed to ensure the validity of the Taylor expansion that is used in the evaluation of the electromagnetic self force about present time $(\tau)$ of position, velocity and acceleration of each element of charge at retarded time during the interval $0<\tau<2 a / c$. With this correction, Eq.(21) is modified to

$\ddot{x}(t)= \begin{cases}0 & , t<0 \\ -\int_{t}^{\infty} f\left(t^{\prime}\right) \frac{d}{d t^{\prime}}\left[e^{-\frac{1}{b} \int_{t}^{t^{\prime}} \frac{d t^{\prime \prime}}{\eta\left(t^{\prime \prime}\right)}}\right] \mathrm{d} t^{\prime} & , t \geq 0\end{cases}$

A simple example helps to clarify the differences between Eq.(21) and Eq.(22). Let $f(t)$ be a constant force that acts during a finite period of time,

$$
f(t)= \begin{cases}0 & , 0<t<t_{0} \\ k & , t_{0}<t<t_{1} \\ 0 & , t>t_{1}\end{cases}
$$

The solution Eq.(21) is given by [9]

$$
\ddot{x}(t)= \begin{cases}k e^{t / b}\left(e^{-t_{0} / b}-e^{-t_{1} / b}\right) & , 0<t<t_{0} \\ k\left[1-e^{-\left(t_{1}-t\right) / b}\right] & , t_{0}<t<t_{1} \\ 0 & , t>t_{1} .\end{cases}
$$

If we assume following form for the correction function $\eta(t)$ :

$$
\eta(t)= \begin{cases}0 & , 0<t<t_{0} \\ \frac{\left(t-t_{0}\right)^{2}}{4 a^{2}} & , t_{0}<t<t_{0}+2 a \\ 1 & , t>t_{0}+2 a\end{cases}
$$

where we suppose that $t_{0}+2 a<t_{1}$, Eq.(22) can be evaluated exactly. We get

$$
\ddot{x}(t)= \begin{cases}0 & , 0<t<t_{0} \\ k\left[1-e^{\frac{4 a}{b}\left(1-\frac{a}{t-t_{0}}\right)-\frac{\left(t_{1}-t_{0}\right)}{b}}\right], & \\ t_{0}<t<t_{0}+2 a & \\ k\left[1-e^{-\left(t_{1}-t\right) / b}\right], & , t>t_{1} . \\ t_{0}+2 a<t<t_{1} & \\ 0 & (26)\end{cases}
$$

. Eq.(26) shows there is no pre-acceleration in the interval $0<t<t_{0}$ before the external force begins to act. In the limit $t \rightarrow t_{0}^{+}$, solution (26) reduces to $\ddot{x}\left(t_{0}\right)=k$, i. e., the acceleration equals the external force per unit mass when the external force is first applied, a result that Yaghjian shows is valid in general. Figure 4 displays the curves of accelerations calculated with Eq.(24) and Eq.(26) for $t_{0}=2, t_{1}=5, a=1$, and $k=0.1$. When $\eta(t)$ reduces to 1 , after $t=t_{0}+2 a$, both solutions agree.

To summarize, the LD equation suffers from two major problems: pre-acceleration and runaway solutions. To have a physically acceptable equation of motion two

approaches have been proposed. In the first, we introduce a correction function multiplying the electromagnetic self-force term in the original LD equation to eliminate the pre-acceleration [8] and impose a boundary condition to eliminate the runaway solution. And in the second by an appropriate procedure we substitute the third order differential LD equation by an effective second order differential equation [7. Rohrlich [6] advocate to take this effective second order equation 


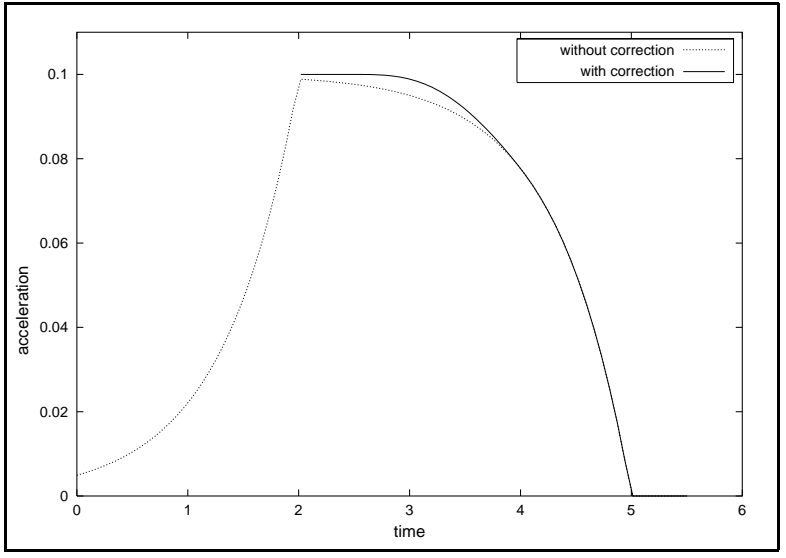

Figure 4: Accelerations calculated with Eq.(24) and Eq.(26) for $t_{0}=2, t_{1}=5, a=$ $1, k=0.1$, (1 unit of length $=2.818 \times 10^{-15}$ $\mathrm{m}, 1$ unit of time $=9.399 \times 10^{-24} \mathrm{~s}$ ). Note the elimination of pre-acceleration when the correction function is included in the AbrahamLorentz equation of motion.

as the right equation for the charged particle motion.

Acknowledgments. D.V. thanks CAPES and P.S.L. thanks FAPESP and CNPQ for financial support.

\section{References}

[1] P.A.M. Dirac, Proc. R. Soc. (London) A167 (1938) 148.

[2] H. Spohn, Europhys. Lett. 50 (2000) 287.

[3] See, for example, E. Zauderer, Partial Differential Equations of Applied Mathematics, 2nd. ed., John Wiley \& Sons, 1989, p. 136.
[4] W.E. Baylis, J. Huschilt, Phys. Rev. D 13 (1976) 3262.

[5] J.M. Aguirregabiria, J. Phys. A: Math. Gen. 30 (1997) 2391.

[6] F. Rohrlich, Phys. Lett. A 283 (2001) 276.

[7] L.D. Landau, E.M. Lifshitz, The Classical Theory of Fields, Butterworth - Heinemann, Oxford, 1975.

[8] A. D. Yaghjian, Relativistic Dynamics of a Charged Sphere, Springer-Verlag, Heidelberg, 1992.

[9] G.N. Plass, Rev. Mod. Phys. 33 (1961) 37.

[10] C. Chicone, S.M. Kopeikin, B. Mashhoon, D.G. Retzloff, Phys. Lett. A 285 (2001) 17. 